\title{
Studies on the development of the claws in new born calves of different breeds and crossbreds using Dual Energy X-Ray Absorptiometry (DXA)
}

\author{
Dedicated to Prof. Dr. Dr. h.c. mult. Ernst Kalm on the occasion of his $65^{\text {th }}$ birthday
}

\begin{abstract}
This study evaluated claw development in 199 calves of different genetic background during the first 70 days of life. Dual Energy X-Ray Absorptiometry (DXA) was found to be a useful tool for this purpose. By the means of DXA, in humans as in animals, body constitution and composition can be examined in vivo. Thus, the whole body or body regions can be analyzed for the content of fat tissue, lean tissue, bone mineral content and bone mineral density. Large differences in claw parameters were observed, depending on the position of the claws, sex and breed, including interaction effects between gender and breeds.

In all four pairs of claws, the medial claws had significantly higher contents of bone mineral than the lateral claws. All fore claws had a higher density than hind claws. Male calves showed significantly higher values than females in all measured parameters.

Comparing breeds and crossbreds, pure German Holsteins had the lowest values in all parameters with exception of the R-value. Within breeds/crossbreds and gender various interactions could be found.

Calves of first lactation cows had lower values than calves of elder cows. Calves with a small birth weight and calves originating of a multiple birth had significantly lower values of the scanned parameters than calves with higher birth weight or monoparous calves.
\end{abstract}

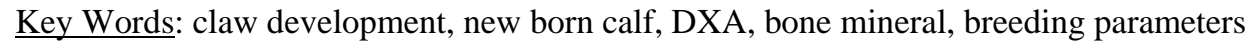

\section{Zusammenfassung}

Titel der Arbeit: Studien zur Entwicklung der Klauen neugeborener Kälber verschiedener Rassen und Kreuzungen mit Hilfe der Dual Energie Röntgen Absorptiometrie (DXA)

Die vorliegende Arbeit bewertet die Klauenentwicklung von 199 Kälbern verschiedener Rassen und Kreuzungen während der ersten 70 Lebenstage. Als Instrument für diese Bewertung wurde die Dual Energie RöntgenAbsorpiometrie genutzt. Mit dieser Methode können an lebenden Menschen oder Tieren Körperkonstitution und Körperzusammensetzung untersucht werden. Auf diese Art können der ganze Körper oder Teile davon auf den Gehalt an mineralisiertem und nichtmineralisierten Gewebe überprüft werden.

Zwischen den gemessenen Klauenparametern wurden große Unterschiede gefunden, die sich aus der Position der Klaue, dem Geschlecht, der Rasse/Kreuzung und aus Effekten zwischen Geschlecht und Rassen ergaben.

An allen vier Gliedmaßen wurden für die medialen Klauen höhere Knochenmineralgehalte gemessen, als für die lateralen. Alle vorderen Klauen hatten eine höhere Knochenmineraldichte als die hinteren Klauen. Männliche Kälber zeigten in allen Parametern signifikant höhere Werte als weibliche

Im Vergleich verschiedener Rassen/Kreuzungen wurden für reine Deutsche Holsteins mit Ausnahme des RWertes die niedrigsten Parameter gemessen. Innerhalb der Rassen und Geschlechter konnten verschiedene Interaktionen beobachtet werden.

Von Kühen der ersten Laktation abstammendeKälber hatten deutlich niedrigere Werte als die Kälber älterer Kühe. Ein niedriges Geburtsgewicht und die Abstammung aus einer Zwillingsträchtigkeit führte zu signifikant niedrigeren Werten der untersuchten Parameter.

Schlüsselwörter: Klauenentwicklung, Kälber, DXA, Knochenmineralisierung, Zuchtwerte 
1. Introduction

Little is known about the development of the bovine claw despite it being the seat of lesions causing severe lameness and losses (HINRICHS et al., 2003). Claw development can be expressed as shape measured linearly, as we did in this paper, using the same experimental group to study the growth and the development of the claw capsule (NÜSKE et al. 2003). Volume, content and density of the content of the capsula ungulae yet have not been investigated in a larger number of growing animals. It is not known at what age the claw reaches maturity. This is of economic importance regarding the timing of first calving and the possibility that overburdening immature claws may have long-term implications for claw health. There is inadequate information regarding the time scale of claw deterioration after the point of maturity. The purpose of this paper is to investigate the use of Dual Energy X-Ray Absorptiometry (DXA) examining the claws in new born calves as a method to develop basic protocols in claw development. By the means of DXA, in humans as in animals, body constitution and composition can be examined in vivo. Thus, the whole body or body regions can be analyzed for the content of fat tissue, lean tissue, bone mineral content and bone mineral density (MITCHELL and SCHOLZ, 1998).

In addition to linear measurements, giving a picture of the claws shape, our intention is to give an impression of the development inside the claws and to look for possibilities to use these parameters for breeding purposes.

Material and methods

2.1. Study sample

Beginning at day 4 post partum (p.p.), four investigations were performed at the Experimental Farm Oberschleissheim within a three weeks interval. Male and female calves of different breeds/crossbreds were measured under light sedation. These six breeds were the two-purpose breed German Fleckvieh (FV), the dairy breed German Holsteins (GH) and F1 crossbred calves of these two breeds. Crossbred calves with Fleckvieh sire and Holstein cow are assigned FV-GH, while crossbreds with Holstein sire and Fleckvieh cow are assigned GH-FV. Following generations of crossbreds with a paramount proportion of Fleckvieh are assigned FVX, following generations with a paramount proportion of German Holsteins are assigned GHX (Table 1). Not all calves could be measured four times depending on health problems or the individual response on sedation.

Table 1

Number of calves within measurement, genetic origin and gender (Anzahl der Versuchstiere nach Messnummer, Abstammung und Geschlecht)

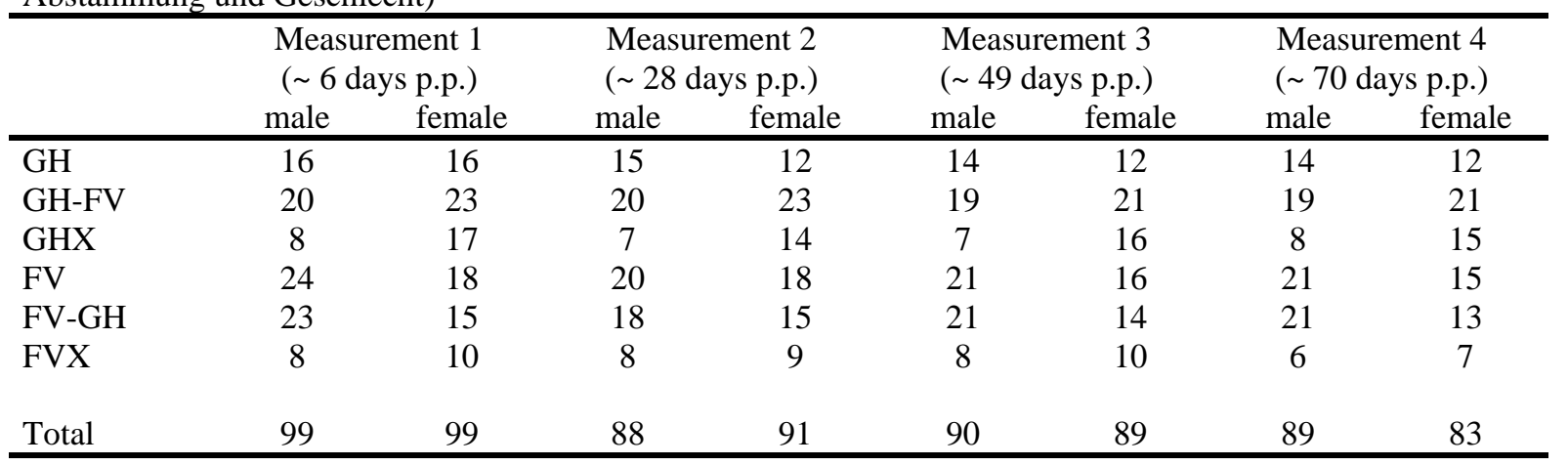


The calves originate from the mating of $9 \mathrm{GH}$ sires and 12 FV sires with totally 52 heifers and 147 cows ( $2^{\text {nd }}$ to $7^{\text {th }}$ lactation). Among all calves were 66 twins (from 33 twin births).

The multiple births originated from $6 \mathrm{GH}, 13 \mathrm{GH}-\mathrm{FV}, 4 \mathrm{GHX}, 8 \mathrm{FV}$ and 2 FV-GH cows. ${ }^{1)}$ For the distribution of all calves within genotype and lactation see Table 2.

Up to the $7^{\text {th }}$ day of life, all calves were fed individually colostrum and housed in single calf boxes on straw mats under outside conditions. From day 8, the calves were housed in groups on straw in a cold stall. They received - depending on age - milk replacer over an automatic milk feeder (Westfalia $\left.{ }^{\circledR}\right)$. In addition, they received ad libitum hay, corn (whole kernels) and on farm produced concentrates (pelleted).

Table 2

Distribution of calves within genetic origin and lactation (Verteilung der Versuchstiere nach Abstammung und Lakationsnummer der Mutterkuh)

\begin{tabular}{llllllll|l}
\hline Lactation & 1 & 2 & 3 & 4 & 5 & 6 & 7 & Total \\
Genotype & & & & & & & & \\
\hline GH & 16 & 17 & 18 & 12 & 5 & 1 & 2 & 71 \\
GH-FV & 11 & 4 & 1 & - & - & - & - & 16 \\
GHX & 3 & 4 & 1 & - & - & - & - & 8 \\
FV & 14 & 31 & 18 & 7 & 13 & 2 & - & -18 \\
FV-GH & 7 & 7 & 2 & 1 & 1 & - & - & 1 \\
FVX & 1 & - & - & - & - & - & - & 199 \\
\hline
\end{tabular}

The measurements were performed under light sedation with Xylazin 2\% (1.5 - 2.5 $\mathrm{ml} / 100 \mathrm{~kg}$ body weight). Body weight was recorded at each measurement day. All parameters were measured with a GE LUNAR DPX-IQ scanner.

Using a Ceriumphotonfilter, polyenergetic $\mathrm{x}$-ray emission can be divided in two energy niveaus (38 keV and $70 \mathrm{keV}$ ). Measurements depend on the differential attenuation of low- and high energy $\mathrm{x}$-rays by fat or other soft tissues and bone. The fat and lean content for each pixel that does not overlie bone is based on the soft tissue attenuation ratio (R-value) being independent of tissue thickness. Division in bone mineral and soft tissue is performed by a software intern treshold operation with bone mineral having the highest attenuation ratio (SCHOLZ, 2002). Theoretically, the Rvalue for bone is 2.8617, for lipids 1.2058 - 1.2333 and for water 1.3572 (PIETROBELLI et al., 1996).

By the help of the LUNAR DPX-IQ software, we defined a region of interest within the scan-image from the tip of the toe up to the fetlock joint of each measured claw.

The parameters being considered were body weight, bone mineral content (BMC), bone mineral density (BMD), bone mineral area, $x$-ray-attenuation coefficient (R), the amount of fat in the measured area (fat\%) and the proportional content of non fatty (lean tissue) and fatty tissue (fat).

\footnotetext{
${ }^{1}$ All animals involved in this study were treated according to the guidelines for care and use of agricultural animals in research.

(Registration number of the Government of Upper Bavaria 211-2531.2-57/2000)
} 


\subsection{Statistical procedure}

A variance analysis was performed using a General Linear Model (Proc GLM, SAS 8.1) with the following structure of fixed effects for all linear claw parameters (SAS/STAT, 1993):

$$
\begin{aligned}
& \mathrm{Y}_{\mathrm{ijklmnop}}=\mu+\mathrm{C}_{\mathrm{i}}+\mathrm{M}_{\mathrm{j}}+\mathrm{O}_{\mathrm{k}}+\mathrm{G}_{\mathrm{l}}+\mathrm{L}_{\mathrm{m}}+\mathrm{B}_{\mathrm{n}}+\mathrm{W}_{\mathrm{o}}+\left(\mathrm{M}_{\mathrm{j}} * \mathrm{O}_{\mathrm{k}}\right)+\left(\mathrm{M}_{\mathrm{j}} * \mathrm{G}_{\mathrm{l}}\right)+\left(\mathrm{G}_{\mathrm{j}} * \mathrm{O}_{\mathrm{k}}\right)+ \\
& \left(\mathrm{M}_{\mathrm{j}} * \mathrm{C}_{\mathrm{i}}\right)+\left(\mathrm{M}_{\mathrm{j}} * \mathrm{G}_{\mathrm{l}} * \mathrm{C}_{\mathrm{i}}\right)+\mathrm{e}_{\mathrm{ijklmno}} \\
& \mathrm{Y}_{\mathrm{ijklmnop}}=\text { Observation } \\
& \mu \quad=\text { population mean } \\
& \mathrm{C}_{\mathrm{i}} \quad \text { = } \text { Claw }(\mathrm{i}=1-8) \\
& \mathrm{M}_{\mathrm{j}} \quad=\text { Number of Measurement }(\mathrm{j}=1-4) \\
& \mathrm{O}_{\mathrm{k}} \quad=\text { Genetic Origin }(\mathrm{k}=1-6) \\
& \mathrm{G}_{\mathrm{l}} \quad \text { Gender }(\mathrm{l}=1-2)
\end{aligned}
$$

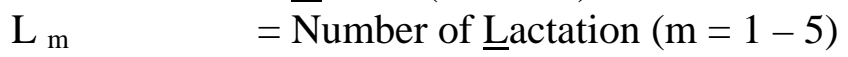

$$
\begin{aligned}
& \mathrm{B}_{\mathrm{n}} \quad=\text { Multiple Birth }(\mathrm{n}=1-2) \\
& \mathrm{W}_{\mathrm{o}} \quad=\text { Birth Weight Class }(\mathrm{o}=1-7) \\
& \mathrm{M}_{\mathrm{j}} * \mathrm{O}_{\mathrm{k}} \quad=\text { Interaction between Number of Measurement and Genetic } \underline{\text { Origin }} \\
& \mathrm{M}_{\mathrm{j}} * \mathrm{G}_{\mathrm{l}} \quad=\text { Interaction between Number of Measurement and } \underline{\text { Gender }}
\end{aligned}
$$

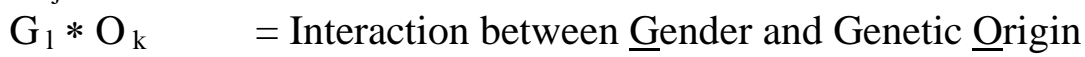

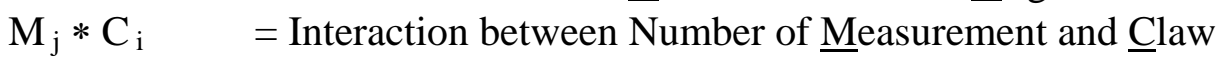

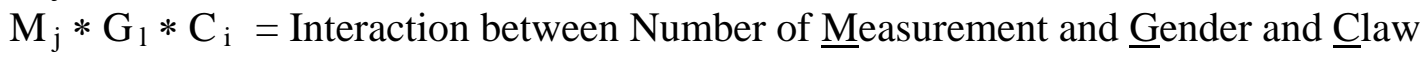

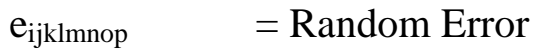

The following figures and tables contain (if not other stated) Least Squares Means (LSM) and corresponding Standard Errors of Estimation (SEE). The significance level was set for $\mathrm{p} \leq 0.05$.

\section{Results}

3.1. Measurement number and claw development

Within the whole study period, the range of all parameters changed severely. As shown in table 3, the difference was significant for every parameter. Body weight increased from 49.333 to $81.1006 \mathrm{~kg}$. Within our region of interest, bone mineral showed an increase of $4.094 \mathrm{~g}$, soft tissue gained $36.32 \mathrm{~g}$. Fat tissue gained $1.75 \mathrm{~g}$, but the percentage of fat within the investigated area decreased for $1.66 \%$.

Table 3

Range of all parameters within the whole study period (Difference was calculated from Least Squares Means of Measurement 1 and 4) (Veränderung aller Parameter innerhalb der gesamten Studie)

$\begin{array}{llllllll}\begin{array}{l}\text { Body } \\ \text { weight } \\ (\mathrm{kg})\end{array} & \begin{array}{l}\mathrm{BMC} \\ (\mathrm{g})\end{array} & \begin{array}{l}\text { Bone } \\ \text { mineral }\end{array} & \begin{array}{l}\text { BMD } \\ \left(\mathrm{g} / \mathrm{cm}^{2}\right)\end{array} & \mathrm{R} & \begin{array}{l}\text { Fat } \\ (\%)\end{array} & \begin{array}{l}\text { Fat tissue } \\ (\mathrm{g})\end{array} & \begin{array}{l}\text { Lean } \\ \text { tissue } \\ (\mathrm{g})\end{array} \\ & & & & & \\ \end{array}$

\begin{tabular}{lllllllll}
\hline Measurem. 1 & $49.333 \pm$ & $10.532 \pm$ & $16.805 \pm$ & $0.613 \pm$ & 1.3668 & $12.41 \pm$ & $16.38 \pm$ & $112.54 \pm$ \\
& 0.254 & 0.064 & 0.064 & 0.002 & \pm 0.0004 & 0.18 & 0.28 & 0.51 \\
Measurem. 4 & $81.106 \pm$ & $14.626 \pm$. & $20.975 \pm$ & $0.690 \pm$ & 1.3704 & $10.75 \pm$ & $18.13 \pm$ & $147.04 \pm$ \\
& 0.269 & 0.068 & 0.067 & 0.002 & \pm 0.0004 & 0.18 & 0.28 & 0.52 \\
\hline Difference & $\mathbf{3 1 . 7 7 3}$ & $\mathbf{4 . 0 9 4}$ & $\mathbf{4 . 1 7 0}$ & $\mathbf{0 . 0 7 7}$ & $\mathbf{0 . 0 0 3 6}$ & $\mathbf{- 1 . 6 6}$ & $\mathbf{1 . 7 5}$ & $\mathbf{3 4 . 5 0}$
\end{tabular}


Significant interactions were found for BMC, BMD, bone mineral area, fat and soft tissue.

BMC had higher values in hind than in fore claws. In all four pairs of claws, the medial claws had significantly higher contents of bone mineral than the lateral claws (Fig. 1).

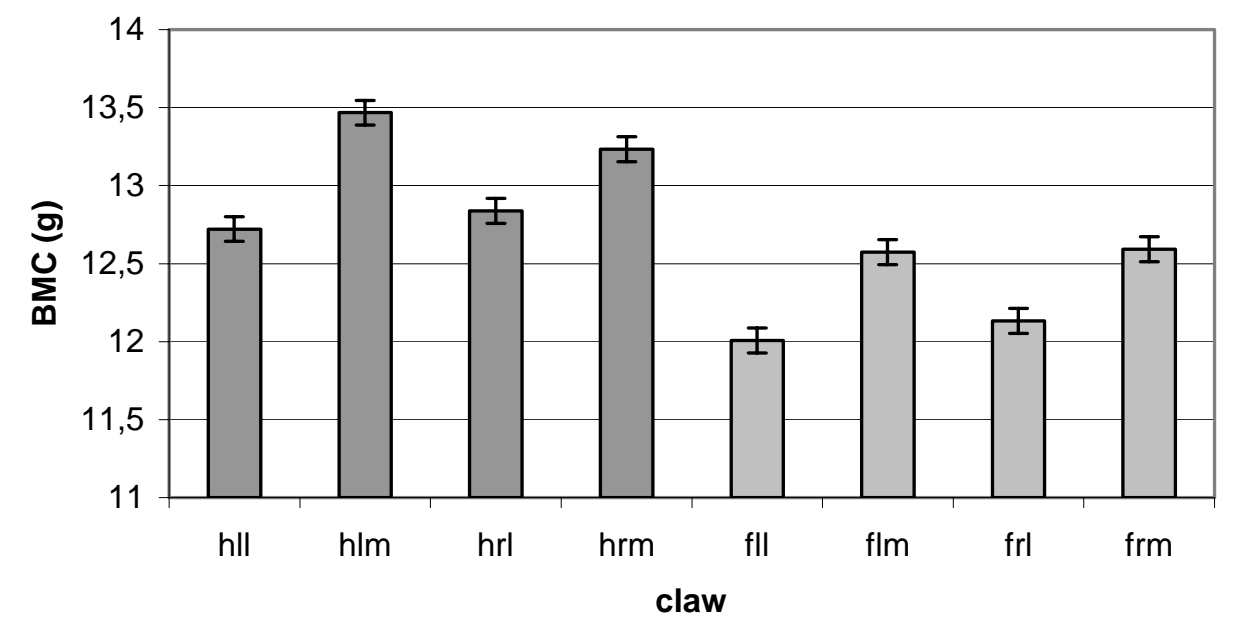

hll - hind left lateral / hind left medial / hrl - hind right lateral / hrm - hind right medial fll - fore left lateral / flm - fore left medial / frl - fore right lateral / frm -fore right medial

Fig. 1: Bone Mineral Content and position of the claw (Knochenmineralgehalt und Klauenposition)

Bone mineral density had an inverse pattern, all fore claws had a higher density than hind claws. Fore medial claws had significantly higher density than lateral claws, hind lateral claws showed higher values in lateral than in medial claws (Fig. 2).

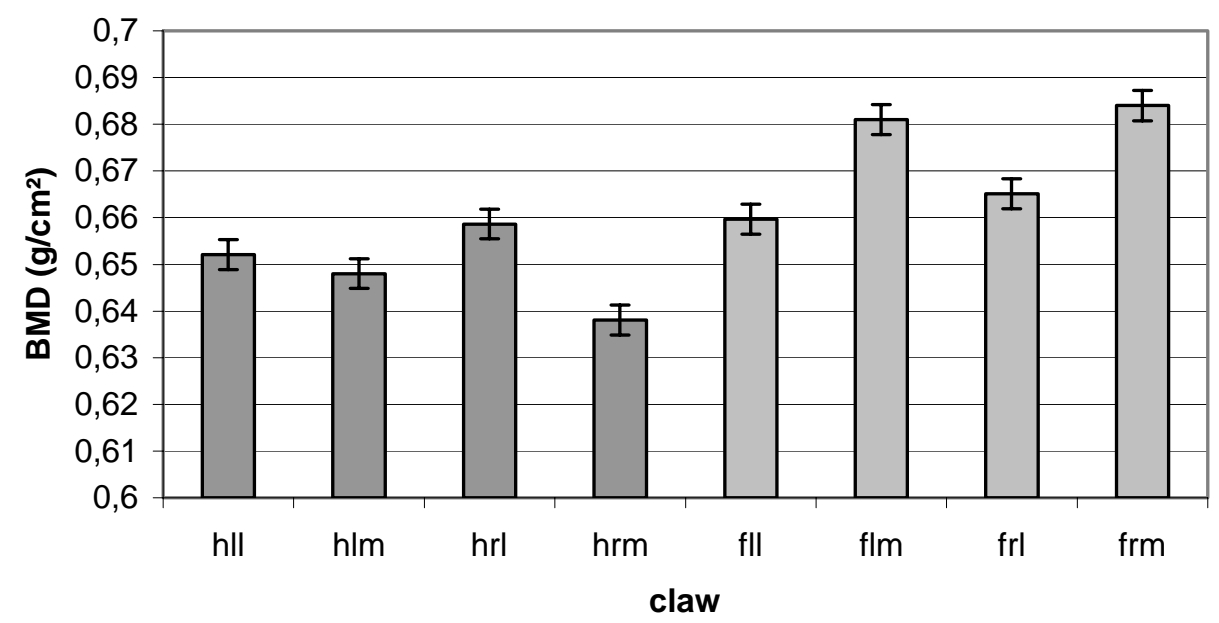

hll - hind left lateral / hind left medial / hrl - hind right lateral / hrm - hind right medial fll - fore left lateral / flm - fore left medial / frl - fore right lateral / frm -fore right medial

Fig. 2: Bone Mineral Density and position of the claw (Knochenmineraldichte und Position der Klaue)

Bone mineral area was larger in hind than in fore claws, all medial claws were larger than lateral claws, in hind claws these values were significantly different (Fig. 3). 


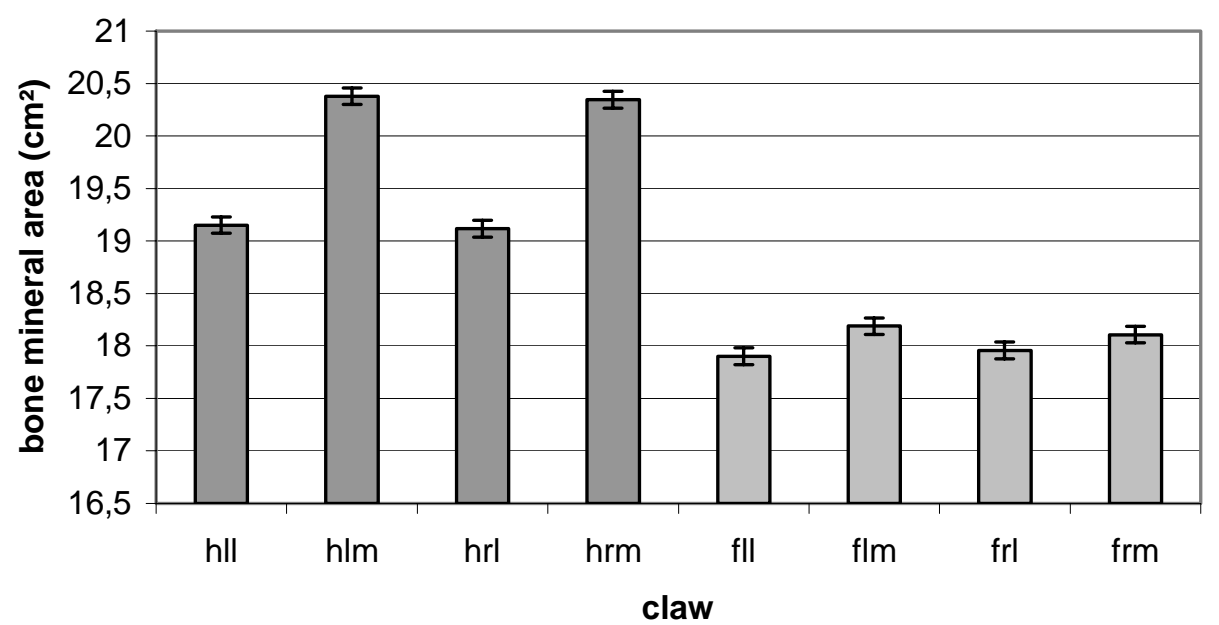

hll - hind left lateral / hind left medial / hrl - hind right lateral / hrm - hind right medial fll - fore left lateral / flm - fore left medial / frl - fore right lateral / frm -fore right medial

Fig. 3: Bone mineral area and position of the claw (Knochenmineral Fläche und Position der Klaue)

Fat and soft tissue showed complex patterns, not allowing a clear statement for lateral or medial, fore and hind claws (Fig. 4). For soft tissue, the amount in fore claws was significantly lower than in hind claws.

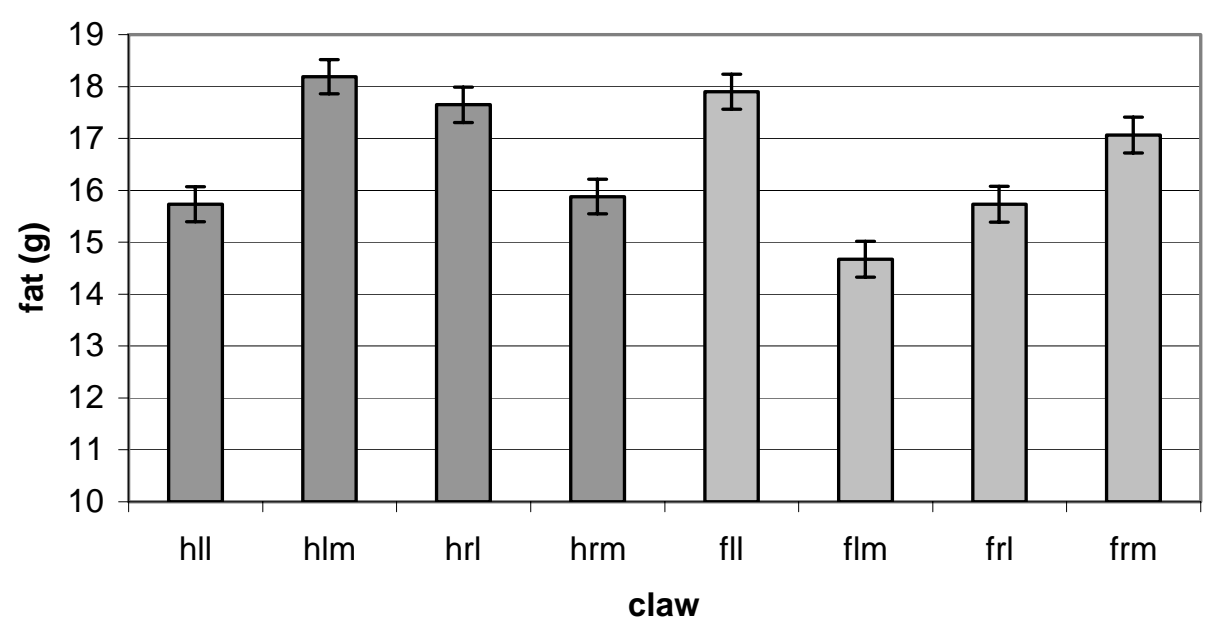

hll - hind left lateral / hind left medial / hrl - hind right lateral / hrm - hind right medial

fll - fore left lateral / flm - fore left medial / frl - fore right lateral / frm -fore right medial

Fig. 4: Fat and position of the claw (Fettgehalt und Position der Klaue)

\subsection{The effect of gender}

All scan parameters except $\mathrm{R}$ value were significantly higher in male than in female calves (Tab. 4).

Investigating the interactions between gender and number of measurement led to some interesting results. BMC and BMD had significantly higher values in male than in female calves for measurement 1 up to measurement 3. While BMC was significantly higher in male than in female calves at the $4^{\text {th }}$ measurement, BMD was higher in 
female than in male calves. (Fig. 5 and 6). Fat percentage decreased from the first to the $4^{\text {th }}$ measurement, male calves having higher values than females (Fig. 7).

Table 4

Scan parameters in male and female calves (Least Squares Mean \pm Standard Error of Estimation *) (Scan Parameter nach Geschlechtern)

\begin{tabular}{lllllllll}
\hline & $\begin{array}{l}\text { Body } \\
\text { weight } \\
(\mathrm{kg})\end{array}$ & BMC $(\mathrm{g})$ & $\begin{array}{l}\text { Bone } \\
\text { mineral } \\
\text { area }\left(\mathrm{cm}^{2}\right)\end{array}$ & $\begin{array}{l}\text { BMD } \\
\left(\mathrm{g} / \mathrm{cm}^{2}\right)\end{array}$ & & $\mathrm{R}$ & $\begin{array}{l}\text { Fat } \\
(\%)\end{array}$ & $\begin{array}{l}\text { Lean tissue Fat } \\
(\mathrm{g})\end{array}$ \\
\hline Male & $64.083^{\mathrm{a}}$ & $12.976^{\mathrm{a}}$ & $19.216^{\mathrm{a}}$ & $0.665^{\mathrm{a}}$ & $1.3686^{\mathrm{a}}$ & $11.53^{\mathrm{a}}$ & $133.06^{\mathrm{a}}$ & $17.53^{\mathrm{a}}$ \\
& \pm 0.223 & \pm 0.056 & \pm 0.056 & \pm 0.002 & \pm 0.0003 & \pm 0.15 & \pm 0.44 & \pm 0.24 \\
Female & $62.234^{\mathrm{b}}$ & $12.414^{\mathrm{b}}$ & $18.570^{\mathrm{b}}$ & $0.656^{\mathrm{b}}$ & $1.3704^{\mathrm{b}}$ & $10.83^{\mathrm{b}}$ & $126.10^{\mathrm{b}}$ & $15.67^{\mathrm{b}}$ \\
& \pm 0.227 & \pm 0.057 & \pm 0.057 & \pm 0.002 & \pm 0.0003 & \pm 0.15 & \pm 0.45 & \pm 0.24 \\
\hline
\end{tabular}

*Least Squares Means with different letters are significantly different $(\mathrm{p} \leq 0,05)$

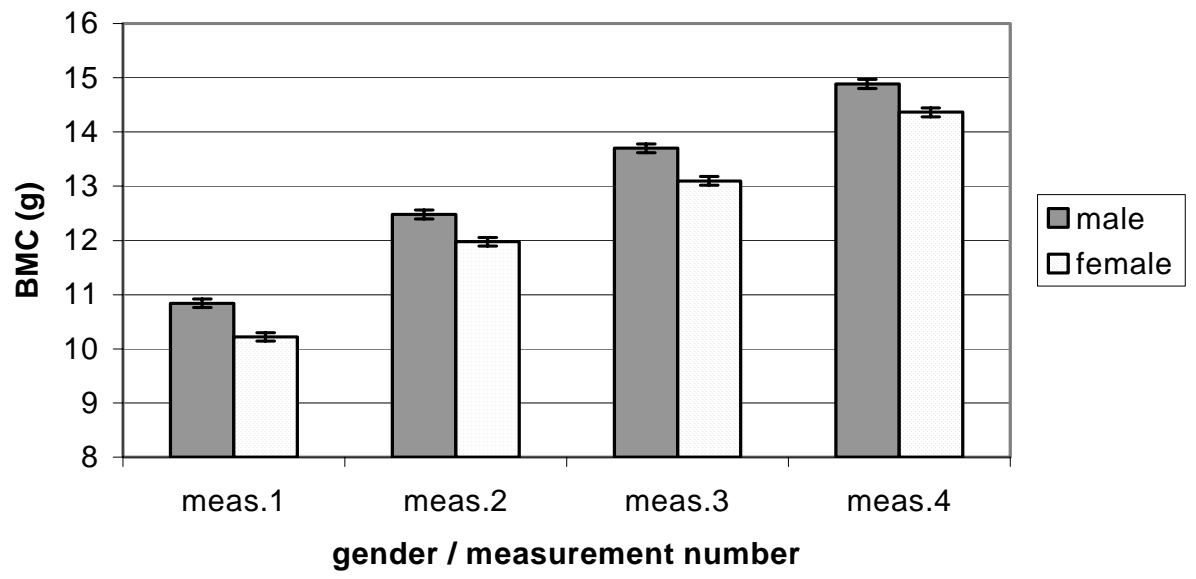

Fig. 5: Bone Mineral Content depending on gender and number of measurement (Knochenmineralgehalt nach Geschlecht und Messnummer)

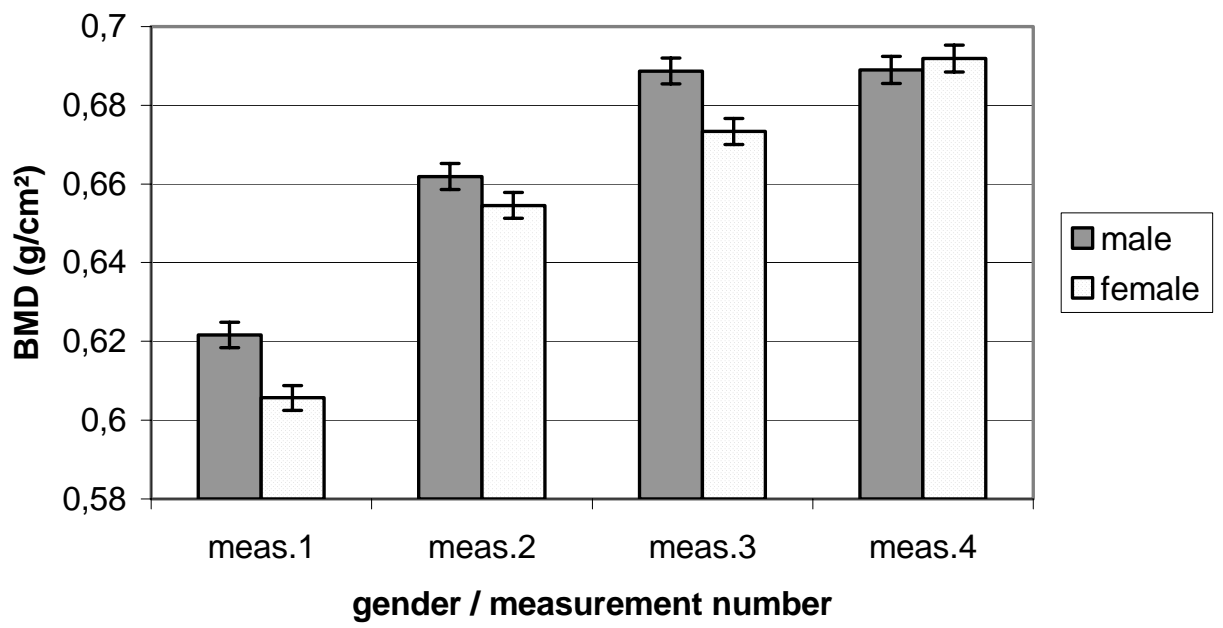

Fig. 6: Bone Mineral Density depending on gender and measurement number (Knochenmineraldichte nach Geschlecht und Messnummer) 


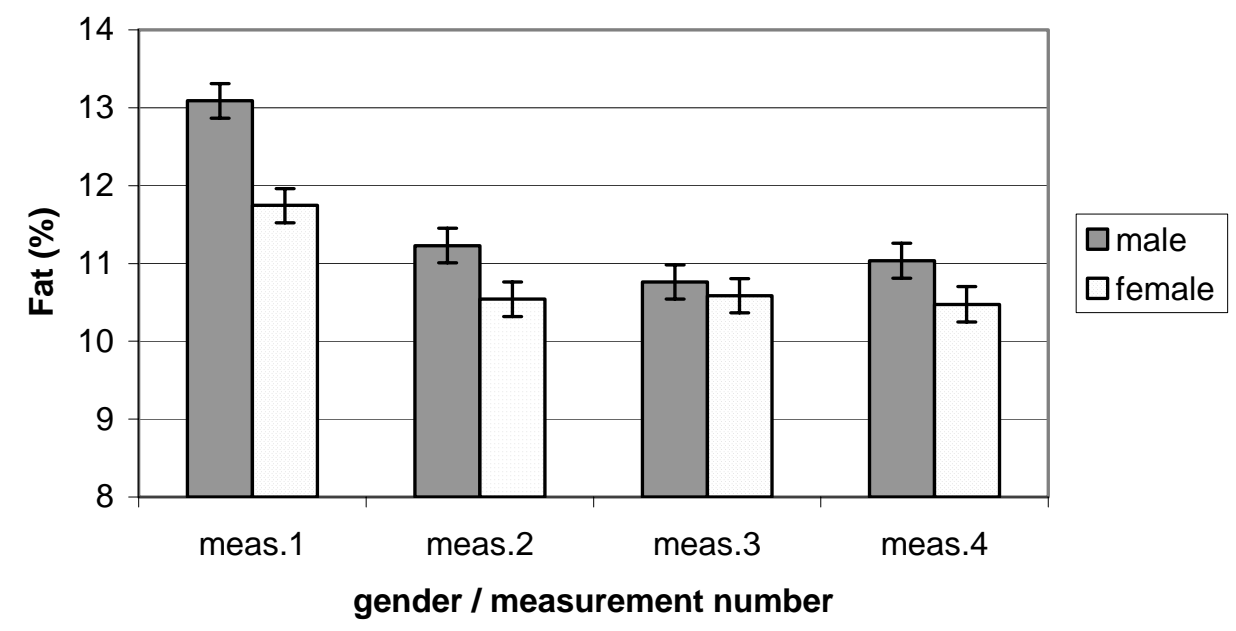

Fig. 7: Fat percentage depending on sex and number of measurement (Fettgehalt nach Geschlecht und Messnummer)

3.4. The effect of breeds and crossbreds

Pure German Holsteins (GH) had the lowest values in all scan parameters with exception of the x-ray attenuation coefficient (Tab. 5).

Table 5

Scan parameters in different breeds/crossbreds (Least Squares mean \pm Standard Error of Estimation*) (Scan Parameter nach Rassen/Kreuzungen)

\begin{tabular}{|c|c|c|c|c|c|c|c|c|}
\hline & $\begin{array}{l}\text { Body } \\
\text { weight } \\
\text { (kg) }\end{array}$ & BMC (g) & $\begin{array}{l}\text { Bone } \\
\text { mineral } \\
\text { area }\left(\mathrm{cm}^{2}\right)\end{array}$ & $\begin{array}{l}\text { BMD } \\
\left.\left(\mathrm{g} / \mathrm{cm}^{2}\right)\right)\end{array}$ & $\mathrm{R}$ & Fat (\%) & $\begin{array}{l}\text { Lean tissue } \\
\text { (g) }\end{array}$ & $\begin{array}{l}\text { Fat } \\
\text { (g) }\end{array}$ \\
\hline \multirow[t]{2}{*}{$\overline{\mathrm{GH}}$} & $61.109^{c}$ & $11.513^{c}$ & $18.157^{c}$ & $0.621^{d}$ & $1.378^{\mathrm{a}}$ & $10.51^{\mathrm{ab}}$ & $123.71^{\mathrm{a}}$ & $14.93^{c}$ \\
\hline & \pm 0.303 & \pm 0.076 & \pm 0.076 & \pm 0.003 & \pm 0.0005 & \pm 0.21 & \pm 0.60 & \pm 0.33 \\
\hline \multirow[t]{2}{*}{ GH-FV } & $64.949^{a}$ & $13.148^{\mathrm{a}}$ & $19.056^{\mathrm{ab}}$ & $0.679^{\mathrm{ac}}$ & $1.3713^{\mathrm{a}}$ & $10.64^{\mathrm{ab}}$ & $131.11^{b}$ & $15.79^{d}$ \\
\hline & \pm 0.270 & \pm 0.068 & \pm 0.068 & \pm 0.002 & \pm 0.0004 & \pm 0.18 & \pm 0.54 & \pm 0.29 \\
\hline \multirow[t]{2}{*}{ GHX } & $62.097^{d}$ & $12.379^{d}$ & $18.599^{d}$ & $0.655^{b}$ & $1.3680^{\mathrm{b}}$ & $11.79^{\mathrm{C}}$ & $124.21^{\mathrm{a}}$ & $17.00^{\mathrm{ab}}$ \\
\hline & \pm 0.345 & \pm 0.088 & \pm 0.086 & \pm 0.003 & \pm 0.0005 & \pm 0.23 & \pm 0.67 & \pm 0.36 \\
\hline \multirow[t]{2}{*}{ FV } & $62.844^{b}$ & $13.069^{a b}$ & $19.056^{\mathrm{ab}}$ & $0.676^{\mathrm{ac}}$ & $1.3687^{b}$ & $11.47^{\mathrm{c}}$ & $132.44^{\mathrm{c}}$ & $17.48^{\mathrm{ab}}$ \\
\hline & \pm 0.269 & \pm 0.068 & \pm 0.067 & \pm 0.002 & \pm 0.0004 & \pm 0.18 & \pm 0.52 & \pm 0.28 \\
\hline \multirow[t]{2}{*}{ FV-GH } & $64.939^{a}$ & $13.154^{\mathrm{a}}$ & $19.266^{\mathrm{c}}$ & $0.671^{\mathrm{c}}$ & $1.3701^{\mathrm{a}}$ & $11.01^{\mathrm{b}}$ & $133.56^{c}$ & $16.61^{\mathrm{a}}$ \\
\hline & \pm 0.297 & \pm 0.075 & \pm 0.074 & \pm 0.003 & \pm 0.0004 & \pm 0.20 & \pm 0.58 & \pm 0.32 \\
\hline \multirow[t]{2}{*}{ FVX } & $63.014^{b}$ & $12.909^{b}$ & $19.222^{\mathrm{bc}}$ & $0.66^{0 \mathrm{~b}}$ & $1.3680^{\mathrm{b}}$ & $11.65^{c}$ & $132.44^{\mathrm{bc}}$ & $17.77^{\text {ab }}$ \\
\hline & \pm 0.353 & \pm 0.089 & \pm 0.088 & \pm 0.003 & \pm 0.0005 & \pm 0.23 & \pm 0.68 & \pm 0.37 \\
\hline
\end{tabular}

*Least Squares Means with different letters are significantly different $(\mathrm{p} \leq 0.05)$

Comparing measurement 1 and four, this clearly can be seen in Figure 8, showing the values of BMC depending on genetic origin and measurement number. Within breeds/crossbreds and gender different interactions exist. For example BMD has complex patterns depending on genetic origin and gender. While male calves generally show higher values than females, in the crossbred FVX, female calves have higher BMD-values than males (Fig. 9). 


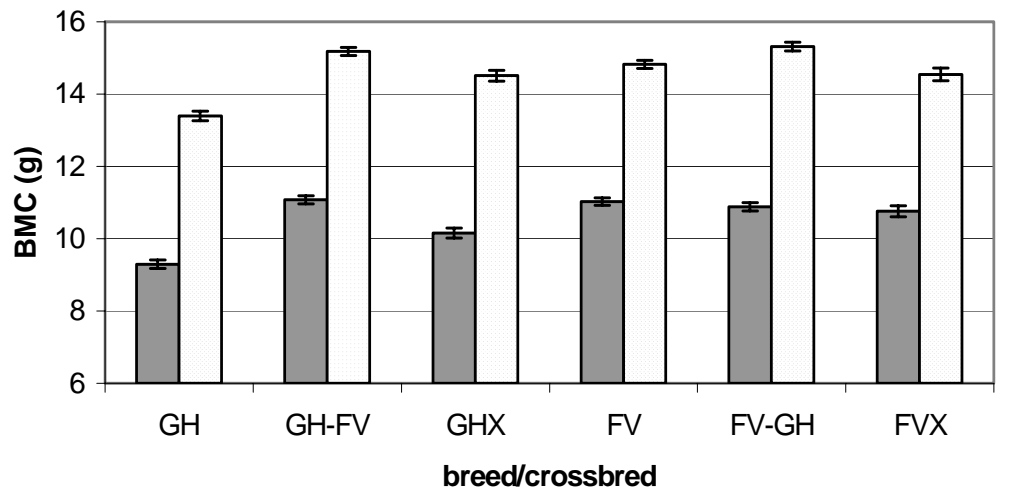

$\square$ measurement 1 measurement 4

Fig. 8: Bone Mineral Content depending on breed/crossbred and number of measurement (Knochenmineralgehalt in Abhängigkeit von Rasse/Kreuzung und Messnummer)

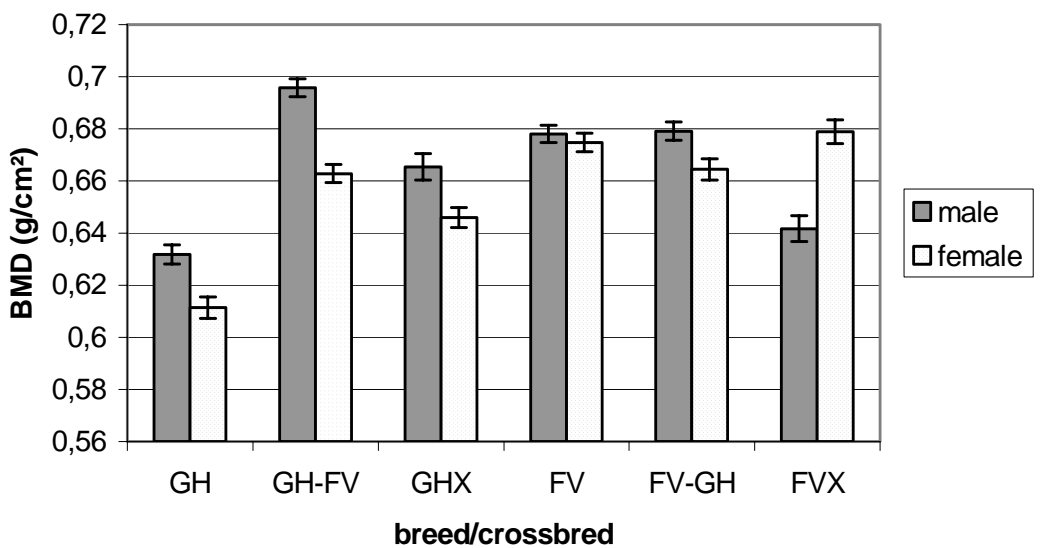

Fig. 9: Bone Mineral Density depending on gender and breed/crossbred (Knochenmineraldichte in Abhängigkeit von Geschlecht und Rasse/Kreuzung)

Table 6

Scan parameters depending on the lactation number (Least Squares Mean \pm Standard Error of Estimation*) (Scan-Parameter in Abhängigkeit zur Lakationsnummer der Mutterkuh)

\begin{tabular}{|c|c|c|c|c|c|}
\hline $\begin{array}{l}\text { Lactation } \\
\text { Parameter }\end{array}$ & 1 & 2 & 3 & 4 & 5 \\
\hline Body weight & $60.955^{d}$ & $63.039^{a}$ & $63.008^{a}$ & $64.873^{b}$ & $63.918^{\mathrm{C}}$ \\
\hline (kg) & \pm 0.282 & \pm 0.229 & \pm 0.281 & \pm 0.365 & \pm 0.304 \\
\hline BMC & $12.000^{\mathrm{c}}$ & $12.936^{\mathrm{a}}$ & $12.747^{b}$ & $13.102^{\mathrm{a}}$ & $12.691^{b}$ \\
\hline (g) & \pm 0.071 & \pm 0.058 & \pm 0.071 & \pm 0.090 & \pm 0.077 \\
\hline Bone mineral & $18.471^{b}$ & $18.998^{\mathrm{a}}$ & $19.055^{\mathrm{ab}}$ & $19.020^{\mathrm{a}}$ & $18.920^{\mathrm{ab}}$ \\
\hline area $\left(\mathrm{cm}^{2}\right)$ & \pm 0.071 & \pm 0.057 & \pm 0.070 & \pm 0.092 & \pm 0.076 \\
\hline BMD & $0.637^{\mathrm{c}}$ & $0.667^{\mathrm{a}}$ & $0.658^{b}$ & $0.676^{\mathrm{d}}$ & $0.664^{\mathrm{a}}$ \\
\hline$\left(\mathrm{g} / \mathrm{cm}^{2}\right)$ & \pm 0.002 & \pm 0.002 & \pm 0.002 & \pm 0.003 & \pm 0.003 \\
\hline \multirow[t]{2}{*}{$\mathrm{R}$} & $1.3712^{\mathrm{a}}$ & $1.3691^{b}$ & 1.3673 & $1.3696^{\mathrm{bc}}$ & $1.3703^{\text {a c }}$ \\
\hline & \pm 0.0004 & \pm 0.0003 & \pm 0.0004 & \pm 0.0005 & \pm 0.0005 \\
\hline Fat & $10.44^{\mathrm{a}}$ & $11.29^{b}$ & $12.07^{\mathrm{c}}$ & $11.14^{\mathrm{b}}$ & $10.96^{\mathrm{ab}}$ \\
\hline$(\%)$ & \pm 0.19 & \pm 0.16 & \pm 0.19 & \pm 0.25 & \pm 0.21 \\
\hline Lean tissue & $127.15^{b}$ & $130.29^{\mathrm{a}}$ & $129.48^{\mathrm{a}}$ & $131.19^{\mathrm{a}}$ & $129.78^{\mathrm{a}}$ \\
\hline (g) & \pm 0.55 & \pm 0.46 & \pm 0.55 & \pm 0.71 & \pm 0.60 \\
\hline Fat & $15.07^{\mathrm{b}}$ & $16.77^{\mathrm{a}}$ & $17.98^{\mathrm{C}}$ & $16.94^{\mathrm{a}}$ & $16.22^{\mathrm{a}}$ \\
\hline (g) & \pm 0.30 & \pm 0.25 & \pm 0.30 & \pm 0.39 & \pm 0.32 \\
\hline
\end{tabular}

* Least Squares Mean with different letters are significantly different $(\mathrm{p} \leq 0,05)$ 
3.5. Effect of the lactation number of the mother

Depending on the lactation number of the mother, Table 6 shows the differences for all parameters including body weight. Calves of first lactation cows had lower values, mostly significant, than calves of elder cows. Overall, the parameters investigated increased from the first to the $4^{\text {th }}$ lactation, then a slight decrease can be recognized.

\section{6. $\quad$ Effect of birth weight}

Birth weight affects scan parameters with exception of the R-value and Fat \% in a linear pattern.Body weight, BMC, BMD and bone mineral area significantly increase with birth weight class (Tab. 7).

Table 7

Scan parameters in different birth weight classes (Least Squares Mean \pm Standard Error of Estimation*) (ScanParameter in Abhängigkeit der Geburtsgewichte)

\begin{tabular}{|c|c|c|c|c|c|c|c|c|}
\hline $\begin{array}{l}\text { Birth } \\
\text { weight }(\mathrm{kg})\end{array}$ & $\begin{array}{l}\text { Body } \\
\text { weight (kg) }\end{array}$ & $\begin{array}{l}\text { BMC } \\
\text { (g) }\end{array}$ & $\begin{array}{l}\text { Bone } \\
\text { mineral area } \\
\left(\mathrm{cm}^{2}\right)\end{array}$ & $\begin{array}{l}\text { BMD } \\
\left(\mathrm{g} / \mathrm{cm}^{2}\right)\end{array}$ & $\mathrm{R}$ & $\begin{array}{l}\text { Fat } \\
(\%)\end{array}$ & $\begin{array}{l}\text { Lean tissue } \\
\text { (g) }\end{array}$ & $\begin{array}{l}\text { Fat } \\
\text { (g) }\end{array}$ \\
\hline \multirow[t]{2}{*}{30} & $51.279^{a}$ & $9.621^{a}$ & $15.114^{\mathrm{a}}$ & $0.626^{\mathrm{ab}}$ & $1.3717^{\mathrm{abc}}$ & $10.19^{\mathrm{ab}}$ & $107.55^{b}$ & $12.67^{\mathrm{d}}$ \\
\hline & \pm 0.527 & \pm 0.133 & \pm 0.132 & \pm 0.005 & \pm 0.0010 & \pm 0.46 & \pm 1.31 & \pm 0.72 \\
\hline \multirow[t]{2}{*}{35} & $56.019^{b}$ & $10.694^{b}$ & $17.102^{b}$ & $0.618^{a b}$ & $1.3693^{\mathrm{abc}}$ & $11.21^{\mathrm{ab}}$ & $118.09^{c}$ & $14.89^{\mathrm{a}}$ \\
\hline & \pm 0.353 & \pm 0.089 & \pm 0.188 & \pm 0.003 & \pm 0.0005 & \pm 0.24 & \pm 0.69 & \pm 0.38 \\
\hline \multirow[t]{2}{*}{40} & $60.713^{c}$ & $11.650^{c}$ & $18.319^{c}$ & $0.632^{\mathrm{a}}$ & $1.3700^{\mathrm{ab}} \pm$ & $11.04^{\mathrm{ab}}$ & $124.61^{d}$ & $15.59^{\mathrm{a}}$ \\
\hline & \pm 0.228 & \pm 0.057 & \pm 0.057 & \pm 0.002 & 0.0003 & \pm 0.15 & \pm 0.44 & \pm 0.24 \\
\hline \multirow[t]{2}{*}{45} & $62.964^{\mathrm{d}}$ & $12.171^{\mathrm{d}}$ & $18.848^{d}$ & $0.643^{c}$ & $1.3693^{c}$ & $11.35^{b}$ & $127.86^{\mathrm{e}}$ & $16.49^{\mathrm{c}}$ \\
\hline & \pm 0.226 & \pm 0.057 & \pm 0.057 & \pm 0.002 & \pm 0.0003 & \pm 0.15 & \pm 0.43 & \pm 0.23 \\
\hline \multirow[t]{2}{*}{50} & $66.339^{\mathrm{e}}$ & $13.249^{\mathrm{e}}$ & $19.864^{e}$ & $0.660^{d}$ & $1.3678^{d}$ & $11.90^{\mathrm{d}}$ & 135.22 & $18.67^{b}$ \\
\hline & \pm 0.327 & \pm 0.083 & \pm 0.082 & \pm 0.003 & \pm 0.0005 & \pm 0.22 & $\pm 0.62^{f}$ & \pm 0.34 \\
\hline \multirow[t]{2}{*}{55} & $70.231^{f}$ & $14.618^{f}$ & $21.100^{f}$ & $0.681^{\mathrm{e}}$ & $1.3680^{\mathrm{cd}}$ & $11.58^{\mathrm{bd}}$ & $146.70^{\mathrm{a}}$ & $19.27^{b}$ \\
\hline & \pm 0.613 & \pm 0.154 & \pm 0.154 & \pm 0.006 & \pm 0.0009 & \pm 0.40 & \pm 1.15 & \pm 0.63 \\
\hline \multirow[t]{2}{*}{60} & $74.566^{\mathrm{g}}$ & $16.863^{g}$ & $21.901^{g}$ & $0.762^{f}$ & $1.3704^{\mathrm{bcd}}$ & $10.99^{a b d}$ & $147.03^{a}$ & $18.60^{\mathrm{b}}$ \\
\hline & \pm 0.925 & \pm 0.233 & \pm 0.232 & \pm 0.009 & \pm 0.0014 & \pm 0.61 & \pm 1.75 & \pm 0.96 \\
\hline
\end{tabular}

* Least Squares Means with different letters are significantly different $(\mathrm{p} \leq 0,05)$

\subsection{Effect of multiple birth}

All parameters in this investigation were significantly higher in monoparous than in pluriparous calves. Mean body weight in monoparous calves was four kg higher than in pluriparous (Tab. 8).

Table 8

Scan parameters in monoparous and pluriparous calves (Least Squares Mean \pm Standard Error of Estimation*) (Scan-Parameter bei Einlings- und Mehrlingskälbern)

\begin{tabular}{lllllllll}
\hline & $\begin{array}{l}\text { Body } \\
\text { weight } \\
(\mathrm{kg})\end{array}$ & BMC (g) & $\begin{array}{l}\text { Bone } \\
\text { mineral } \\
\text { area }\left(\mathrm{cm}^{2}\right)\end{array}$ & $\begin{array}{l}\text { BMD } \\
\left(\mathrm{g} / \mathrm{cm}^{2}\right)\end{array}$ & R & Fat (\%) & $\begin{array}{l}\text { Lean } \\
\text { tissue }(\mathrm{g})\end{array}$ & Fat (g) \\
\hline pluriparous & $61.195^{\mathrm{a}}$ & $12.222^{\mathrm{a}}$ & $18.537^{\mathrm{a}}$ & $0.646^{\mathrm{a}}$ & $1.3700 \pm$ & 11.06 & $126.80^{\mathrm{a}}$ & $16.11^{\mathrm{a}}$ \\
calves & \pm 0.325 & \pm 0.082 & \pm 0.081 & \pm 0.003 & 0.0005 & \pm 0.22 & \pm 0.64 & \pm 0.35 \\
monoparous & $65.122^{\mathrm{b}}$ & $13.169^{\mathrm{b}}$ & $19.249^{\mathrm{b}}$ & $0.674^{\mathrm{b}}$ & $1.3690 \pm$ & 11.30 & $132.36^{\mathrm{b}}$ & $1^{\mathrm{b}}$ \\
calves & \pm 0.208 & \pm 0.052 & \pm 0.052 & \pm 0.002 & 0.0003 & \pm 0.14 & \pm 0.41 & \pm 0.22 \\
\hline
\end{tabular}

* Least Squares Means with different letters are significantly different $(\mathrm{p} \leq 0,05)$

4. Discussion and Conclusion

Measurements of bone mineral density of the claw region in hind and fore claws give a possibility in judgment of skeleton growth and bone density, which has not yet been 
examined. Investigation of a large number of calves of different breeds and crossbreds and both genders shows, that DXA is suited for the judgment of limbs under genetic aspects.

DXA results change rapidly with the number of measurement, age and body weight All parameters grew, except fat and fat $\%$.

This can be explained by the existence and vanishing of the fetal cushion, which still covers the claw for the first weeks after birth and then disappears. The mass of fat decreases from scan one to scan two and slightly begins to increase with scan 3 . That means, between three and seven weeks p.p. the fetal cushion is diminished by walking. The percentage of fat drops permanently, a sign for strong growth in mineralized and non fatty tissue.

As in the linear claw measurement section (NÜSKE et al., 2003), the first point of interest within DXA-results was aimed at the relations between hind and fore, medial and lateral claws. Bone mineral content was higher in medial claws than in their partner claws. Hind claws generally had higher amounts of bone mineral than fore claws (Fig. 1).

The relations changed when focussing on bone mineral density (Fig. 2). Fore claws showed higher amounts of mineral $\left(\mathrm{g} / \mathrm{cm}^{2}\right)$ than did hind claws. Hind lateral and fore medial claws had a higher density in bone mineral than the partner claws.

Here it is possible to consider weight bearing rules influencing bone development. From adult cattle it is known that fore medial and hind lateral claws have to bear more weight than fore lateral or hind medial claws (VAN DER TOL et al., 2002). Assuming that these rules also account for calves, a higher body weight could influence early development in the described way. Even the higher density in fore claws can be explained in higher load. The fore half of the body takes about $60 \%$ of the whole body weight.

Soft tissue as sum of fat and lean tissue follows the pattern of mineralized tissue (BMC). Hind claws in general have a higher amount oft soft tissue as fore claws. The medial claws dominate the lateral. In fore claws there is no symmetry for soft tissue. For fat, no difference could be found between hind and fore claws, lateral and medial claws. To explain the relations between mineralized and soft tissue, it can be assumed, that the bigger claws are, the more mineralized bone and soft tissue they have.

Analyzing all scan data under the aspect of differences in genders, as expected, brought higher amounts in all parameters for male calves, except the R-value. $\mathrm{R}$, the $\mathrm{X}$-ray-attenuation coefficient for soft tissue is highly correlated ( $\mathrm{r}>0.99$ ) with fat. Low amounts of fat lead to a high R-value. In this investigation the amount of fat in male calves is significantly higher than in females (male $17.35 \pm 0.24 \mathrm{~g}$ / female 15.67 $\pm 0.24 \mathrm{~g}$ ). Therefore $\mathrm{R}$ is higher in female calves (male $1.3686 \pm 0.0003 /$ female $1.3704 \pm 0.0003)$.

$\mathrm{BMC}$ and BMD as described above grow rapidly within the period studied. Comparing breeds and crossbreds, pure Fleckvieh (FV) or crossbreds with Fleckvieh reached higher values for all parameters with exception of the R-value than pure German Holsteins. In their analysis of in vivo body composition of new born calves using DXA, HAMPE et al. (2004) found significant differences of nearly all selected parameters for purebred German Holstein calves. Nevertheless, there are also significant differences within different parameters in all breeds or crossbreds. This leads to the assumption, that there must be a different construction of the claws in 
different breeds/crossbreds, such as a different size or structure of the fatty cushion inside the claw.

By including the effect of gender within breeds, the same strong differences result for all parameters as described above in respect to claws within genders. For example, the amount of BMD in female calves of all breeds or crossbreds is significantly lower than in males with exception of the crossbred FVX, where male calves have lower values than females.

These results show -- as was demonstrated in the results of linear claw measurements - that there is no clear way to judge claw development depending on different breeds or crossbreds without considering genders. Generally, the pattern of claw development depends on gender, genetic origin and on the parameter of interest itself.

Another effect important for the claw development of the calf is the lactation number of the mother. All parameters were higher in calves of elder cows compared to calves of younger cows. Assuming that higher amounts in all parameters are comparable to better claw soundness, calves of elder cows (up to $5^{\text {th }}$ parity) should have an advantage in fundamental "foot health" compared to calves from younger cows.

The influence of different birth weight on the development of the claw could be demonstrated. This could lead to the assumption, that calves with higher birth weight have better claws than calves with lower weights. Indeed we do not yet know, whether bigger claws are better in soundness, this has to be investigated in longer terms, comparing early claw development to mature claws.

DXA scanning gives new aspects of claw development inside the capsula ungulae and even provides information regarding the content of fat in combination with mineralized bone tissue. Nevertheless, it must be recognized, statements to the fatty cushion depend severely on the age of the calf and need further investigation. RAEBER (2000) assumed that load and age influence fat content and fatty acid composition of the fatty cushion within the digit. However in the study of RAEBER (2000) only digits of female cattle have been examined. The own results show, that there also is an influence of gender, breed/crossbred, and even of gender within breeds/crossbreds concerning the development of the digital (fatty) cushion.

Linear measurements and DXA-scanning can be used to characterize early claw development. Both methods combined give a high amount of information on claw structures and growth of horn, which has not been investigated before.

We showed that there are large differences in claw parameters depending on the claws themselves, being fore or hind, medial or lateral claws. As stated above, there are differences between genders, among breeds and crossbreds, and even within breeds and genders. Thus, claw development can not be described in general. Breeds or crossbreds and gender must be considered in programs concerning breeding or selection of lines. DISTL (1999) used similar measurement parameters as we did in the study of NÜSKE et al. (2003) for improvement of foot and leg soundness in dairy cattle. He concluded also to consider both genders in the improvement of foot and leg traits.

The age of the mother cow has a strong influence on the calf's claw development. This aspect underlines the importance of life-production, not only production on a high level for a few lactations.

Nevertheless, the central factor in describing claw development is, that up to now it is not really clear, which parameter is ideal for selection and health evaluation. As seen 
in the results section, the same parameter in different breeds or genders can get different dimensions. There are no strait results which would generally confirm relations between single parameters and diseases or disorders of the claw. Though high efforts in this direction have been undertaken by claw trimmers and veterinarians in order to develop improved trimming methods, stronger activities should be aimed at programs analyzing data on measurable parameters, which fit better the needs of a more scientific knowledge based diagnosis of claw and leg problems.

DISTL, O.:

\section{References}

Zucht auf ein gesundes Fundament beim Milchrind. (Breeding for soundness of feet and legs in dairy cattle) Züchtungskunde 71 (1999), 446 - 458

HAMPE, J.; NÜSKE, S.; SCHOLZ, A.M.; FÖRSTER, M.:

Analysis of in vivo body composition of new born calves using dual energy x-ray absorptiometry (DXA). W207, J. Anim. Sci. 82, Suppl. 1, J. Dairy Sci. 87, Suppl. 1, Poult. Sci. 83 (2004) Suppl. 1: 365

HINRICHS, D.; KUHLMANN, T.O.; STAMER, E.; KALM, E.:

Genetische Analyse von Klauenmaßen aus der Eigenleistungsprüfung von Jungbullen. Arch. Tierz., Dummerstorf 46 (2003) 2, 155-165

MITCHELL, A.D.; SCHOLZ, A.M.; CONWAY, J.M.:

Body composition analysis of small pigs by dual-energy x-ray absorptiometry. J. Anim. Sci., 7 (1998), 2392-2398

NÜSKE, S.; SCHOLZ, A.M.; FÖRSTER, M.:

Studies on the growth and the development of the claw capsule in new born calves of different breeding lines using linear measurements. Arch. Tierz., Dummerstorf 46 (2003) 6, 547-557

PIETROBELLI, A.; FORMICA, C.; WANG, Z.; HEYMSFIELD, S.B.:

Dual-energy x-ray absorptiometry body composition model: review of physical concepts., American Physiological Society, (1996), E941-E951

RAEBER, M.:

Das Ballenpolster beim Rind - ein Beitrag zur funktionellen Anatomie der Klaue. (The digital cushion SAS / STAT in cattle - a contribution to the functional anatomy of the claw) Diss. Med.vet. 2000; Zürich, CH.

User's Guide Volume 2, GLM-VARCOMP, Version 6 Fourth Edition, by SAS Institute Inc., Cary, NC, USA, 1993; 891-996

SCHOLZ, A.M.:

In-vivo-Methoden zur Analyse von Muskelstoffwechsel und Körperzusammensetzung beim Schwein unter besonderer Berücksichtigung genetischer Einflüsse. Habilitationsschrift der Tierärztlichen Fakultät der Ludwig-Maximilians-Universität München, (2002)

http://epub.ub.uni-muenchen.de/archive/00000418/T

VAN DER TOL, P.P.J.; METZ, J.H.M.; NOORDHUIZEN-STASSEN, E.M..; BACK, W.; BRAAM, C.R.; WEIJS, W.A.:

Pressure distribution on the bovine claw while standing, in Proceedings. $12^{\text {th }}$ Int. Symp. Lameness in Ruminants 2002; 202-205

Received: 2005-01-21

Accepted: 2005-06-15

Author's addresses

Dr. STEFAN NÜSKE, JULIA HAMPE, Priv. Doz. Dr. Dr. habil. ARMIN SCHOLZ

Lehr- und Versuchsgut der Tierärztlichen Fakultät der Ludwig-Maximilians-Universität

Hubertusstr. 12

85764 OBERSCHLEIßHEIM / GERMANY

E-Mail: stefan.nueske@lmu.de

Univ.-Prof. Dr. Dr. habil. MARTIN FÖRSTER

Institut für Tierzucht und Allgemeine Landwirtschaftslehre

der Ludwig-Maximilians-Universität

Veterinärstr.13

80805 MÜNCHEN / GERMANY 